\title{
Large gauge invariance in non-Abelian finite temperature effective actions
}

\author{
S. Deser \\ Department of Physics, Brandeis University, Waltham, Massachusetts 02454 \\ L. Griguolo \\ Dipartimento di Fisica, Universitá di Parma, INFN, Gruppo Collegato di Parma, Parco Area Viale delle Scienze 7/A, 43100 Parma, Italy \\ D. Seminara \\ Dipartimento di Fisica, Universitá di Firenze, INFN, Sezione di Firenze, Via G. Sansone 1, 55125, Sesto Fiorentino, Florence, Italy
}

(Received 12 December 2002; published 27 March 2003)

\begin{abstract}
We analyze large gauge invariance in the non-Abelian, finite temperature context and its physical consequences for $D=3$ effective actions. After briefly reviewing the structure of bundles and large gauge transformations that arise in nonsimply connected 3-manifolds and gauge groups, we discuss their connections to Chern-Simons terms and Wilson-Polyakov loops. We then provide an invariant characterization of the "Abelian" fluxes encountered in explicit computations of finite temperature effective actions. In particular we relate, and provide explicit realizations of, these fluxes to a topological index that measures the obstruction to global diagonalization of the loops around compactified time. We also explore the fate of, and exhibit some everywhere smooth, large transformations for nonvanishing index in various topologies.
\end{abstract}

DOI: 10.1103/PhysRevD.67.065016

PACS number(s): 11.10.Wx, 11.15.Bt, 11.30.Er, 11.30.Rd

\section{INTRODUCTION}

Finite temperature gauge physics differs significantly from its nonthermal counterpart. The geometrical features related to the presence of $S^{1}$-compactified Euclidean time considerably complicate the zero-temperature picture. Already for Abelian effective actions (Dirac determinants) generated by integrating out charged fermions in $D=3$, a number of unexpected phenomena emerge [1,2] through the interplay between "large" gauge invariance requirements and the topological properties of nonsimply connected spacetimes. Through careful regularization, it was possible to deal with the usual array of "global" properties, such as spectral asymmetries (characteristic of odd dimension), parity anomalies, preservation of large gauge invariance and the role of (generalized) Chern-Simons (CS) terms. In the process, we were able to exhibit very general "Fourier" representations of these $U(1)$ actions such that the large gauge transformations (LGT) arising at finite temperature were properly expressed with respect to the corresponding, nonperturbative, Ward identities. In turn, this led to gaugeinvariant expansions of the effective actions useful for both large and small fermion masses, where the anomalies and ambiguities could also be analyzed.

The object of the present work is the natural but nontrivial extension of the above analysis to non-Abelian theories, for various-simply connected or not-internal symmetry groups such as $S U(2)$ and $S O(3)$. The basic issue remains that of large gauge invariance, but with the added nonAbelian gauge complication. It is known that (as in the Abelian case) massive fermions in a background non-Abelian gauge field at $T \neq 0$ induce, at the one-loop level, a CS term whose coupling parameter is continuous in temperature [3-5]. Therefore, at generic $T$, this parameter cannot have the discrete value required for invariance under LGT. On the other hand we could expect $[1,6,7]$, from the Abelian analy- sis, that at finite temperature an infinite number of terms is induced in the effective action in such a way that large gauge invariance is restored, even though it would not be manifest at any finite perturbative order. The above statement can be easily proved by studying the relevant Dirac determinant using $\zeta$-function regularization, a manifestly gauge-invariant method providing a well-defined geometrical tool for discussing global properties. While the spacetime aspects of the Dirac determinants (now augmented by the color index entries) in this formalism are very similar to the Abelian case, so the various series expansions presented in Ref. [1] carry over formally unchanged, this procedure unfortunately gives insight neither into the mechanism through which the perturbative series organizes itself nor into the nonperturbative information, such as holonomies, indices and fluxes, needed to explain the global invariance. Indeed, we shall see that significant nonperturbative changes arise precisely for configurations that involve a novel index.

The feasibility of an explicit description of the large gauge invariance properties in the Abelian case is related to the peculiar structure of the $U(1)$ gauge group. Abelian gauge transformations with nontrivial winding number exist only at finite temperature, where the CS action jumps discontinuously by a nonvanishing boundary term when magnetic flux is present on the underlying two-dimensional manifold. Conversely, it was possible to show that the only degree of freedom of the gauge field transforming significantly under LGT was the flat connection wrapping around $S^{1}$. Large gauge invariance is precisely the statement that the effective action must be periodic in the flat connection, implying in this way an additional finite Ward identity. Moreover, this critical, topological, degree of freedom affects the parity violating (PV) part of the action only when accompanied by magnetic flux on the corresponding two-dimensional manifold. In the Abelian case, therefore, the nontrivial effects of LGT are unavoidably linked to the simultaneous presence of 
a magnetic flux and of a flat connection. ${ }^{1}$ Our general considerations were illustrated by explicit integration in presence of some specific, physically nontrivial, field configurations $^{2}$ [1] (see also Ref. [8]). While the topological complications of Abelian theory arose in a very specific and "rigid" way, disappearing in the (topologically trivial) limit of zero temperature, non-Abelian LGT and some of their consequences persist at $T=0$, as illustrated by the wellknown quantization [11] of the tree-level CS coefficient. At one loop and zero temperature, the nontrivial topological part of the PV action is entirely governed by a gauge invariant functional of the field, usually denoted as $\eta(0)$, that is neither local nor continuous. It can be explicitly computed with the help of the Atiyah-Patodi-Singer theorem [12] and consists of two parts: a continuous local functional given by the CS action plus a nonlocal, discontinuous, contribution given by a topological index [13]. Large gauge invariance is maintained through a cancellation between the two. While, for massless fermions, this is the only PV contribution, for massive fermions, new (but harmless) structures can be generated: their form in the limit of small and large mass is discussed in Ref. [1]. A direct zero-temperature perturbative computation of the PV effective action produces a properly normalized CS term that respects large gauge invariance in the familiar sense: the quantized nature of the CS coefficient is preserved.

For massive fermions, when the temperature is turned on, however, the very same expansions start, discontinuously, to generate $T$-dependent CS coefficients exactly as in the Abelian case, requiring a combined, thermal plus gauge, description. In the easiest case, when the gauge group is, like $S U(N)$, simply connected, the basic topological properties are unaltered; at the same time the presence of a nontrivial $S^{1}$ and the explicit form of the perturbative results suggests that the zero mode of the time component $A_{0}$ will again play a special role. To be more precise, the (untraced) WilsonPolyakov loop (WPL) around Euclidean time is the important new ingredient, just as it was in the Abelian case. Unfortunately, in the non-Abelian case, flat connections do not behave simply under LGT and it is not immediately obvious what kind of fluxes could be coupled there, since as we will review [in the $S U(N)$ case] the bundle structure is trivial. At the same time, the explicit example of Ref. [14], the perturbative computations of Ref. [15] and the derivative expansions of Ref. [9] suggest that, at least for vanishing $\boldsymbol{E}$, the effective actions bear a strong resemblance to the Abelian ones, requiring also a field endowed with an apparent magnetic flux. We will start our analysis by examining to what

\footnotetext{
${ }^{1}$ The above picture, developed in Ref. [1], is itself a natural extension of one in zero space dimension [6], the important new feature being the magnetic flux.

${ }^{2}$ Our computations were mainly performed with vanishing electric field $\boldsymbol{E}$ purely in order to permit analytical results, since $\boldsymbol{E}$ plays no role in preserving large gauge invariance: Indeed, later constructions with $\boldsymbol{E} \neq 0$ using improved derivative expansions [9] or partially resummed perturbation theory [10] confirmed our conclusions.
}

extent the thermal Abelian mechanism can be embedded in the more complicated non-Abelian setting. In the process, we will encounter a new (in this context) topological index, associated with the obstruction to diagonalizing WPL globally, and playing the role of a generalized magnetic flux. ${ }^{3}$ Moreover, we shall find that a class of LGT can be studied explicitly, for specific field configurations, within a particular gauge fixing; the new topological index is also pivotal to this analysis. Although our embedding is completely general, only in the case of vanishing $\boldsymbol{E}$ does it provide an exhaustive account of the gauge invariance problem: for generic field configurations, it must be accompanied by other mechanisms to deal with the nonflat part of $A_{0}$, i.e., the electric sector. We believe, however, that the LGT properties of the magnetic sector are always controlled in the above manner.

Our paper is arranged as follows: in Sec. II, we set out some basic facts about the topology of internal gauge groups, mappings into spaces of the form $S^{1} \times$ compact Riemann surfaces of arbitrary genus, the structure of the CS term and its finite temperature transformation properties. In particular, we try to extend our earlier, purely three-dimensional, Abelian analysis of the CS coefficient's quantization at finite $T$ to the non-Abelian $S U(N) / Z_{N}$ without going through a fourdimensional embedding argument [20]. We show how, for these nonsimply connected groups, the zero-temperature condition given by the third homotopy group may be modified due to the presence of a nontrivial first homotopy group. In the process, we present and motivate an explicit, simple, smooth and periodic LGT on $S^{1} \times S^{2}$, followed later by one on $S^{1} \times T^{2} \sim T^{3}$. In Sec. III, we turn our attention to WPL, whose analysis was equally central in the Abelian case, because it carries the global gauge invariant information. We note that, while this gauge invariant information is of course contained in its eigenvalues, diagonalizing WPL is, in general, not a smooth operation: this quantity, viewed as a map from the base manifold into the gauge group, might not be globally diagonalizable. Using the formalism of Ref. [19], we associate an index to this topological obstruction and show (by going to a suitable, "diagonal," gauge) that magnetic fluxes appear along the Cartan directions, despite the vanishing of the original bundle's first Chern class. In Sec. IV, we explicitly construct the static temporal gauge $\dot{A}_{0}=0$ in order to analyze the structure of the potentials. We discover a class of LGT preserving this gauge condition when the WPL has a nontrivial index, and transforming the WPL holonomy exactly as for the $U(1)$. Using the above ingredients in Sec. V, we treat configurations with (for simplicity) vanishing electric field along the lines of Ref. [1], the role of magnetic flux being played here by an array of gaugeinvariant magnetic projections related to the above index. A final section summarizes our results and outlines some open problems.

\footnotetext{
${ }^{3}$ This index has already appeared in $\mathrm{QCD}_{4}$ in the discussion of the Abelian projection and of the related 't Hooft-Polyakov monopoles [16-18]. It was, in fact, used to give a topological gauge invariant characterization of this family of solutions. In three dimensions, its role has been also discussed in Ref. [19].
} 


\section{LGT AND TOPOLOGICAL CHARGES}

\section{A. $D=3$ bundles}

Let us first review some basic facts about Euclidean thermal gauge fields on 3 -spaces of the form $\mathcal{M}=S^{1} \times \Sigma_{g}$, where $\Sigma_{g}$ is a compact Riemann surface of genus $g$. The circle $S^{1}$, parametrized by the Euclidean time, $T$, has circumference $\beta=1 / \kappa T$.

In the following, we shall focus on principal $G$ bundles having $\mathcal{M}$ as basis and $G$ as structure group; their topological classification is more involved than for $T=0$. There, one usually compactifies Euclidean three-space on $S^{3}$, the threesphere. Now principal $G$ bundles over $S^{3}$ are classified by $\pi_{2}(G)$ [21], the second homotopy group of $G$; however $\pi_{2}(G)=0$ for any Lie group and hence, in the usual $S^{3}$ picture, the bundle associated with the gauge fields is automatically trivial. The situation changes for more general three-manifolds $\mathcal{M}$, since $G$ bundles are now classified by elements of the cohomology group $H^{2}\left(\mathcal{M}, \pi_{1}(G)\right)$ [22]. We see that nontrivial topological charges could arise when nonsimply connected groups are taken into account; the commonly considered $S U(N)$ case is, instead, still trivial, since $\pi_{1}[S U(N)]=0$. If $G=U(N)$ then $\pi_{1}(G)=\mathbb{Z}$ and the bundle is classified by an element of $H^{2}(\mathcal{M}, \mathbb{Z})$, which may be identified with the first Chern class. At finite temperatures, where $\mathcal{M}=S^{1} \times \Sigma_{g}$ is the relevant base manifold, we find

$$
H^{2}\left(S^{1} \times \Sigma_{g}, \mathbb{Z}\right)=\mathbb{Z}^{2 g+1},
$$

upon using the explicit form of the first Betti number of $\mathcal{M}$, $b_{1}(\mathcal{M})=2 g+1$, and the absence of torsion [23,24]. This clearly generalizes the situation of $N=1$ and $g=0$ (the 2 -sphere), by allowing for (several) magnetic fluxes. [Gauge groups other than $S U(N)$ may also effectively arise in simple situations, for example when adjoint fermions are coupled to $S U(N)$ gauge fields.] Another interesting case is $G=S O(N)$; then $\pi_{1}(G)=\mathbb{Z}_{2}$ and the bundle is classified using $H^{2}\left(\mathcal{M}, \mathbb{Z}_{2}\right)$, which may be identified with the second Stiefel-Whitney class. Using Poincaré duality and some basic properties of homology theory (see for example Ref. [24]), one can compute

$$
H^{2}\left(S^{1} \times \Sigma_{g}, \mathbb{Z}_{2}\right)=Z_{2}^{2 g+1} \text {. }
$$

A third simple example is given by taking $G=S U(N) / Z_{N}$ : in this case one can show along the same lines that

$$
H^{2}\left(S^{1} \times \Sigma_{g}, Z_{N}\right)=Z_{N}^{2 g+1} .
$$

The above classifications are interesting because they exhaust the possible topological sectors of finite temperature $D=3$, providing the analogue of the instanton charges for the gauge groups considered. They will help us understand the permissible values of the CS coefficient and the transformation properties of the CS action itself. At finite temperature, one must specify the boundary conditions on $S^{1}$ : the gauge connections can always be chosen periodic,

$$
A_{\mu}(t+\beta, x)=A_{\mu}(t, x) .
$$

Fermions can be taken to be antiperiodic,

$$
\psi(t+\beta, x)=-\psi(t, x)
$$

if they are in the fundamental representation, or antiperiodic up to a global $\mathbb{Z}_{n}$ transformation (i.e., up to an element of the center), if in the adjoint representation. Therefore the effective gauge group is $S U(N) / Z_{N}$, which allows nontrivial topological sectors.

\section{B. LGT on nonsimply connected manifolds and groups}

We have seen that the presence of a nontrivial $\pi_{1}(\mathcal{M})$ can change topological structure; the natural problem now is to understand how LGT are changed when the base manifold (and possibly the gauge group) is not simply connected. For general $\mathcal{M}$ and $G$, under the simplifying assumption of dealing with trivial bundles, ${ }^{4}$ LGT are classified by the set of the homotopy classes $[\mathcal{M}, G]$ of continuous maps from $\mathcal{M}$ into $G$. In the zero-temperature case, when $\mathcal{M}$ is $S^{3}$ we simply have $[\mathcal{M}, G]=\pi_{3}(G)$, leading to the usual classification in terms of integer winding numbers [26]. For $S U(N)$, we know that $\pi_{3}[S U(N)]=\mathbb{Z}$. The general case is more involved: the homotopy classes $[\mathcal{M}, G]$ are labeled by two topological winding numbers $\left(W_{1}, W_{2}\right)$. The primary, $W_{1}$, and the secondary, $W_{2}$, are elements of the following cohomology groups,

$$
W_{1} \in H^{1}\left(\mathcal{M}, \pi_{1}(G)\right), \quad W_{2} \in H^{3}\left(\mathcal{M}, \pi_{3}(G)\right) .
$$

These topological charges are the obstructions to deforming a generic map $U$ into a constant one. While this finer classification is not really essential for $S U(N)$, whose $\pi_{1}$ vanishes, the primary winding number does become relevant for nonsimply connected groups like $U(N)$ or $S O(N)$. Moreover, for any compact and orientable $\mathcal{M}$ it can be shown [23] that

$$
H^{3}(\mathcal{M}, \mathbb{Z})=\mathbb{Z}
$$

so the structure of LGT for $S U(N)$ is basically unchanged at finite temperature.

We next display the promised LGT example in $S^{1} \times S^{2}$, and (for simplicity) $S U(2)$ :

$$
\begin{aligned}
U_{n}(t, \theta, \phi) & \equiv u(t) \hat{U}_{n}(t, \theta, \phi) \\
& \equiv \exp \left(-i \frac{\pi}{\beta} t \sigma_{3}\right) \exp \left(i \frac{\pi}{\beta} t \boldsymbol{\omega}_{n} \cdot \boldsymbol{\sigma}\right) \\
& =\exp \left(-i \frac{\pi}{\beta} t \sigma_{3}\right)\left[\cos \left(\frac{\pi}{\beta} t\right) I+i \sin \left(\frac{\pi}{\beta} t\right) \boldsymbol{\omega}_{n} \cdot \boldsymbol{\sigma}\right]
\end{aligned}
$$

\footnotetext{
${ }^{4}$ For a nontrivial bundle $E$ the classification of LGT is equivalent to that of $\operatorname{Map}_{G}(E, A d(G))$ (see Ref. [25]). Fortunately, we do not need to study this more complicated question.
} 
In terms of the two-sphere angles $(\theta, \phi)$, the components of the unit 3-vector $\omega_{n}$ are $\omega_{n}^{1}(\theta, \phi)=\sin \theta \cos (n \phi), \omega_{n}^{2}(\theta, \phi)$ $=\sin \theta \sin (n \phi)$ and $\omega_{n}^{3}(\theta, \phi)=\cos \theta$. A similar transformation $^{5}$ was obtained, in a different context, in Ref. [27]; there it was noted that $\hat{U}_{n}$ alone was antiperiodic, but that it could be made periodic simply by composing it with the "small" antiperiodic $u(t)$ that (being angle independent) cannot alter the winding in $\hat{U}_{n}$. This $\hat{U}_{n}$ is just a square root of the well-known periodic ansatz later introduced independently in Ref. [4], which is why it produces all windings, but at the price of antiperiodicity. [The original, periodic, ansatz covered only even windings.] A simple computation confirms that the contribution from $u(t)$ to the winding integrand is a total divergence whose integral vanishes on the sphere. We notice that for $n=0$ the representative is not the identity but a particular, nontrivial, small gauge transformation. It is possible to generalize the above construction to $S U(N)$ by embedding the $S U(2)$ example into $S U(N)$ : the antiperiodicity of $\hat{U}$ is replaced by "periodicity" up to an element of the center $\mathbb{Z}_{N}$ and $u(t)$ by a similar small transformation. In Sec. III we will present an example with different topology, the torus $T^{3}$.

Returning to the classification problem, let us first contrast the above treatment of $S U(N)$ with that of $U(1)$ : For the latter, only $W_{1}$ is relevant $\left(\pi_{1}[U(1)]=Z\right.$ Z), because its $\pi_{3}$ vanishes. [At finite temperature, Abelian LGT therefore appear, whose effects on the quantization of the CS coupling constant and on the definition of the CS action have already been mentioned.] Here we simply write the result for generic $\mathcal{M}$, easily derivable from Ref. [23]:

$$
H^{1}(\mathcal{M}, \mathbb{Z})=(\mathbb{Z})^{2 g+1} .
$$

The gauge groups $U(N>1)$ and $S O(N>3)$, instead, experience the combined effect of both winding numbers:

$$
\begin{gathered}
{[\mathcal{M}, U(N)]=W_{1} \oplus W_{2}=(\mathbb{Z})^{2 g+1} \oplus \mathbb{Z},} \\
{[\mathcal{M}, S O(N)]=W_{1} \oplus W_{2}=\left(\mathbb{Z}_{2}\right)^{2 g+1} \oplus \mathbb{Z} .}
\end{gathered}
$$

The case of $S O(3)$ is special: not all possible primary and secondary winding numbers separately label LGT, but only those having a particular relation. In Ref. [23] it was shown that $W_{1}$ and $W_{2}$ must satisfy $\left(W_{1}\right)^{3}=W_{2} \bmod 2$ as a relation in $H^{3}\left(\mathcal{M}, Z_{2}\right)$ : For $\mathcal{M}=S^{1} \times \Sigma_{g}$, it is not difficult to prove that the above condition can be satisfied trivially since $\left(W_{1}\right)^{3}=0$ and one gets

$$
[\mathcal{M}, S O(3)]=\left(\mathbb{Z}_{2}\right)^{2 g+1} \oplus 2 \mathbb{Z}
$$

\footnotetext{
${ }^{5}$ Any other large transformation $\bar{U}_{n}$ will differ from Eq. (2.8) by a small one. One recent example is that of Ref. [28], which was obtained from the usual $T=0$ form [11], $\bar{U}_{n}^{L}(T=0)$ $=\exp \left[n \pi i \boldsymbol{y} \cdot \boldsymbol{\sigma} / \sqrt{\boldsymbol{y}^{2}+\lambda^{2}}\right]$, by compactifying time through the mapping $t \rightarrow \tau \equiv \tan (\pi t / 2 \beta)$. Here $\boldsymbol{y} \equiv(t, \boldsymbol{x})$ and $\lambda$ is an arbitrary constant cutoff. However, as noted in Ref. [28], the price of this mapping is that [in contrast with Eq. (2.8)] the resulting $\bar{U}_{n}^{L}$ is not analytic.
}

where the second term on the right-hand side simply realizes the condition $W_{2} \bmod 2=0$ : only even secondary winding numbers appear in the classification of LGT for $S O(3)$.

\section{Quantization of CS coefficient on nonsimply connected groups and manifolds}

While the systematic analysis of $D=3$ LGT [23] was originally motivated by studying the vacuum structure of four-dimensional gauge theories, their relevance to $D=3$ physics was realized by analysis of CS theory [11], which we review briefly. When defined on a trivial $G$ bundle over $\mathcal{M}$, the CS action has the form

$$
S=2 \pi k I_{C S}=\frac{k}{4 \pi} \int_{\mathcal{M}} d^{3} x \epsilon^{\alpha \mu \nu} \operatorname{Tr}\left[A_{\alpha} \partial_{\mu} A_{\nu}-\frac{2 i}{3} A_{\alpha} A_{\mu} A_{\nu}\right] .
$$

The behavior of CS under gauge transformations,

$$
A_{\mu}^{U}=U^{-1} A_{\mu} U+i U^{-1} \partial_{\mu} U,
$$

is given by

$$
\begin{aligned}
I_{C S}\left[A^{U}\right]= & I_{C S}[A]+\mathcal{W}[U] \\
& -\frac{i}{8 \pi^{2}} \int d^{3} x \epsilon^{\alpha \mu \nu} \partial_{\alpha} \operatorname{Tr}\left[A_{\mu} U^{-1} \partial_{\nu} U\right],
\end{aligned}
$$

with

$$
\mathcal{W}[U]=\frac{1}{24 \pi^{2}} \int_{\mathcal{M}} d^{3} x \epsilon^{\alpha \mu \nu} \operatorname{Tr}\left[U^{-1} \partial_{\alpha} U U^{-1} \partial_{\mu} U U^{-1} \partial_{\nu} U\right] .
$$

Since the bundle structure is trivial, the total derivative term can be neglected (our manifolds having no boundary); for $S U(N), \mathcal{W}[U]=W_{2}[U]$ measures the secondary winding number or topological degree of $U$. This in turn leads to the integer quantization condition on the coupling constant $k$, since the phase, $S$, is the relevant physical object at quantum level. Of course when $\pi_{1}(G) \neq 0$, the situation may change because the primary winding number can also enter for nontrivial $G$ bundles. In particular, the CS term itself is not well defined when the bundle is nontrivial: the $A_{\mu}$ in Eq. (2.12) are not globally defined as one-forms on $\mathcal{M}$ and the action is patch dependent by virtue of Eq. (2.14). [Here the total derivative contributions must also be taken into account.] The simplest, but physically relevant, realization of this possibility was actually in finite temperature Abelian theory $[1,2,29,30]$, where the nontrivial bundle structure forced an analogous quantization requirement. The total derivative term in Eq. (2.14) contributes when LGT (necessarily of primary type) are performed in presence of topological charges (the analogue of instantons). As a result, we noted that, for the CS action to be well defined, a doubling of the naive 
quantization condition was required. A general approach to the problem was proposed in Ref. [20] by defining the CS action as

$$
S=\frac{k}{4 \pi} \int_{\mathcal{B}} d^{4} x \epsilon^{\alpha \beta \mu \nu} \operatorname{Tr}\left[F_{\alpha \beta} F_{\mu \nu}\right]
$$

where $\mathcal{B}$ is some 4-manifold (or more generally 4-complex) whose boundary is $\mathcal{M}$ and the $G$ bundle has been extended over $\mathcal{B}$. Now it is the $\mathcal{B}$-independence of $S$ that forces $k$ to be an integer, but demanding that the $G$ bundle be extendable poses further restrictions on $k$. We argue here that these restrictions, consistent with our quantization rule in the Abelian case, can also be understood as coming from LGT within the purely three-dimensional approach, at least for $\mathcal{M}=S^{1} \times S^{2}$. To show this, consider $S U(N) / Z_{N}$ : we have shown above that nontrivial bundles can be present, while existence of LGT can also be easily inferred, since now the $S U(N)$ gauge functions need be periodic only up to a $\mathbb{Z}_{N}$ transformation. A particularly explicit realization can be offered for $S O(3)$, equivalent to $S U(2) / Z_{2}$, using the adjoint representation of $S U(2)$, with generators $\left(\tau_{a}\right)_{b c}=i \epsilon_{a b c}$. LGT on $\mathcal{M}$ are labeled by $\mathbb{Z}_{2} \oplus 2 Z$ and are explicitly realized by [27]

$$
\begin{aligned}
& U_{0,2 n}(t, \theta, \phi)=\exp \left(-i \frac{2 \pi}{\beta} t \tau_{3}\right) \exp \left(i \frac{2 \pi}{\beta} t \boldsymbol{\omega}_{n} \cdot \tau\right), \\
& U_{1,2 n}(t, \theta, \phi)=\exp \left(i \frac{2 \pi}{\beta} t \boldsymbol{\omega}_{n} \cdot \tau\right) .
\end{aligned}
$$

We must remark that the $U(1)$ factor in the first of these two classes of transformations is actually a large transformation by itself: it cannot be contracted continuously to unity; only its square can. Its primary winding number is, indeed, 1. Since the second factor also has primary winding number 1 , their product possesses a vanishing $W_{1}$. Recall that $W_{1}$ takes values in $Z_{2}$.

Therefore, the nontrivial $\mathbb{Z}_{2}$ classes are related to a nontrivial loop around $S^{1}$, similar to the LGT of $U(1)$ : at $n$ $=0$ it is more convenient to use a simpler representative of the nontrivial class, depending only on $t$,

$$
\mathcal{U}_{1}(t)=\exp \left(-i \frac{2 \pi}{\beta} t \tau_{3}\right) .
$$

In the following we will work (for general $N$ ) in the fundamental representation, realizing the quotient at the level of gauge functions, periodic up to the particular element of $Z_{N}$ that is identified with the primary winding number. The basic transformation is

$$
\mathcal{U}_{1}(t)=\exp \left(-i \frac{2 \pi}{N \beta} t H\right),
$$

where $H$ is the diagonal, traceless, $N \times N$ matrix with entries $(1,1, \ldots, 1,1-N)$; the elements of the other classes are obtained by taking all powers up to $N-1$. We have now constructed the representatives for primary windings number (at genus zero the classes are in correspondence with $Z_{N}$ ). Next we construct the nontrivial $S U(N) / Z_{N}$ bundles on $S^{1} \times S^{2}$ : as in the $U(1)$ case, one can think of them as coming from monopolelike configurations on the sphere. Nontrivial connections on $S^{2}$ are characterized by a nontrivial $\mathbb{Z}_{N}$ holonomy around, say, the north pole: a simple way to obtain this is to fix the transition functions, labeled by the various elements of $Z_{N}$, and taken at $\theta=\pi / 2$ for definiteness, to be

$$
G_{m}(\phi)=\exp \left(-i \frac{2 \pi m}{N \beta} \phi H\right), \quad m=0,1 \ldots N-1 .
$$

We promote $G_{m}$ to be the transition function of the full $S U(N) / Z_{N}$-principal bundle over $S^{1} \times S^{2}[m$ being the number associated to the different bundle structures, according to Eq. (2.3)]. From the three-dimensional point of view $G_{m}$ is now seen to be the transition function at the intersection of two patches covering $\mathcal{M}$, having toroidal topology (we have implicitly assumed that our transition functions could be chosen time independent). To construct the CS action in this nontrivial case, we can resort to a patch by patch definition:

$$
I_{C S}(A)=I_{C S}^{X_{1}}\left(A_{1}\right)+I_{C S}^{X_{2}}\left(A_{2}\right),
$$

where $X_{1}, X_{2}$ are two solid tori with oppositely oriented boundaries $T^{2}, A_{1}, A_{2}$ being the expressions for the gauge connections (now globally defined on $X_{1}, X_{2}$ ) with boundary values related by the transition function $G_{m}$. As in the Abelian case, the definition (2.21) must be augmented by a term depending explicitly on the transition function, in order to be independent of the particular local trivialization of the bundle (see Refs. [31,29] for the cohomological meaning of these terms). Our proposed generalization of Eq. (2.21) is

$$
\begin{aligned}
I_{C S}(A)= & I_{C S}^{X_{1}}\left(A_{1}\right)+I_{C S}^{X_{2}}\left(A_{2}\right)+\hat{\mathcal{W}}[G] \\
& +\frac{i}{8 \pi^{2}} \int_{T^{2}} d^{2} x \epsilon^{i j} \operatorname{Tr}\left[G \partial_{i} G^{-1} A_{1 j}\right],
\end{aligned}
$$

where $\hat{W}[G]$ is the Wess-Zumino-Witten-Novikov (WZWN) action associated to $G$. The origin of Eq. (2.22) can be understood by recalling Eq. (2.14). It can be checked that for trivial bundles it coincides with the CS action, when written in terms of globally defined connections, and that it reproduces the Abelian result. The definition of the WZWN action implies that the CS coupling parameter $k$ must be even for $N$ even, and an arbitrary integer for $N$ odd, according to the general analysis for $S U(N) / Z_{N}$ presented in Ref. [32]. Gauge invariance further restricts $k$ : let us compute the gauge variation of Eq. (2.22) under $\left(\mathcal{U}_{1}\right)^{s} \equiv \mathcal{U}_{s}(s=1,2 \ldots N-1)$, the LGT with nonvanishing primary winding number, represented as in Eq. (2.19). Since the $\mathcal{U}_{s}$ commute with the transition function $G_{m}$, they are globally defined automorphisms of the bundle. We obtain 


$$
\delta I_{C S}=\frac{1}{8 \pi^{2}} \int_{T^{2}} d^{2} x \epsilon^{i j} \operatorname{Tr}\left[G_{m} \partial_{i} G_{m}^{-1}\left(\mathcal{U}_{s}^{-1} \partial_{j} \mathcal{U}_{s}-\mathcal{U}_{s} \partial_{j} \mathcal{U}_{s}^{-1}\right)\right]
$$

Using the explicit form of $H$, this variation reduces to

$$
\delta I_{C S}=m s \frac{N-1}{N} .
$$

From the extreme case, $m=s=1$, we learn that $k$ must be a multiple of $N$ in order for the quantum phase to be well defined. In Ref. [20] the same problem was examined, following the four-dimensional route and using algebraic geometrical techniques; our more down-to-earth approach can reproduce that result for $N$ odd, but for $N$ even we apparently miss a factor 2 (the quantization rule appearing in Ref. [20] is $k=2 N$ for $N$ even). One possible explanation may be that an extra factor $1 / 2$ is needed in the normalization of the CS term for $N$ even: it is well known $[32,33]$ that WZWN theory needs this extra factor in its definition, when quotiented by $Z_{N}$ for $N$ even. If the CS action experienced the same change we would find full agreement with Ref. [20]; in any case, the general $N$ dependence displayed by our approach indicates that a purely three-dimensional definition is indeed possible.

\section{FLUXES AND THEIR CHARACTERIZATIONS}

\section{A. General framework}

For finite $T$, understanding how the invariance of the effective action under LGT is explicitly realized can be difficult. In the Abelian case, we singled out the flat connection (effectively WPL) wrapping around $S^{1}$ as the carrier of this information, leading to the basic requirement that the effective action be periodic, i.e., have "Fourier" form in WPL. A major role in that analysis was also played by fields with nonvanishing Chern class. (In fact, most of the examples in the Abelian literature exploit this property to generate a candidate CS term in the effective action.) For $S U(N)$, the interplay between periodic time, LGT, and fluxes appears to be weakened: unlike in the Abelian case, LGT survive in the zero-temperature limit and there are no bundles with nonvanishing first Chern class. Therefore the generic non-Abelian picture cannot simply mimic the Abelian one-some new mechanism must be introduced. Nevertheless, in the following, we show that there is a vast class of non-Abelian fields with features common to those of the Abelian analysis and for which one can define apparently Abelian, but fully gauge invariant, fluxes.

As stated, the additional Abelian information carried by the flat connection is encoded in the WPL about $S^{1}$. It is natural to expect that the same quantity will also describe some topological properties of the effective action, such as its behavior under LGT. Here, as we shall see, the WPL is related to a new topological number, whose role is very similar to that of magnetic flux. To illustrate this unexpected connection, we must first introduce some results about maps from a two-manifold into $S U(N)$. In fact, from the mathematical point of view, the (untraced) WPL defined by

$$
\Omega(\beta, \boldsymbol{x})=P \exp \left(-i \int_{0}^{\beta} d t^{\prime} A_{0}\left(t^{\prime}, \boldsymbol{x}\right)\right) \equiv \Omega
$$

is a map from the space manifold, say $S^{2}$, into $S U(N)$. Despite the fact that the $\pi_{2}$ of $S U(N)$ vanishes, we can associate to the applications from $S^{2}$ into the group an integervalued topological index [19] that measures the obstruction to diagonalization of $\Omega$ by globally defined transformations. This integer will play the role of a generalized magnetic flux. (This is not a novel idea: it was indeed widely exploited in $D=4$ to study the invariant meaning of magnetic monopoles and magnetic defects in the gauge where $A_{0}$ is time independent and diagonal [17] and in particular to discuss the fate of LGT in the Hamiltonian approach [18] to $\mathrm{QCD}_{4}$.)

Following Ref. [19], we shall review the invariant characterization of the gauge connections for which this index does not vanish. ${ }^{6}$ We confine our analysis to regular WPL, which are dense in the space of all possible maps. An element $g$ in a group $G$ is regular if the set of elements commuting with $g$ has dimension of the maximal torus $T^{m}$ of $G$. [In $S U(N)$, this statement is equivalent to saying that the eigenvalues of $g$ are nondegenerate.] Our problem may then be formulated as follows: Can a given smooth regular map $g: \Sigma \rightarrow G$ be written as

$$
g=h^{-1} s h
$$

where $s: \Sigma \rightarrow T^{m}$ and $h: \Sigma \rightarrow G$ are smooth and globally defined maps? The answer to this question cannot be always affirmative, as the following classic example shows. Consider the $S U(2)$ element

$$
g \equiv i \hat{\boldsymbol{r}} \cdot \boldsymbol{\sigma}, \quad \hat{\boldsymbol{r}}^{2}=1 .
$$

Since $\hat{\boldsymbol{r}}^{2}=1$ is also a sphere, we can regard $g$ as an application between two $S^{2}$, the second one being the equator of $S U(2)$. To any such application $g$, we can associate a topological winding number given by

$$
n(g)=-\frac{1}{32 \pi} \int_{S^{2}} \operatorname{Tr}(g[d g, d g]) .
$$

Clearly $n(g)$ vanishes identically if $g$ can be diagonalized smoothly. On the other hand, $n(g)$ is equal to 1 for Eq. (3.3), as a simple calculation in polar coordinates shows; thus we must conclude that the above $g$ cannot be smoothly diagonalized. However, when we consider $g$ as a matrix-valued field defined on a bundle, conjugation to the maximal torus, i.e., its diagonalization, acquires the meaning of performing a gauge transformation. Limiting the admissible gauge transformations just to the globally defined ones is too restrictive; the natural geometrical requirement is (as for the gauge connections) rather that they be definable chart by chart, respect-

\footnotetext{
${ }^{6}$ The relevance of this class of fields in $D=3$ physics was already underlined in Ref. [19], where these configurations were shown to be crucial to the path integral solution of CS theory and to the derivation of the Verlinde formula.
} 
ing the cocycle conditions on the overlaps of different charts. ${ }^{7}$ In the following, we will diagonalize $g$ in this wider arena, finding the new index in the process.

First, we must understand how to keep track of the information encoded in $n(g) \neq 0$ if we allow nonsmooth transformations. To this end, observe that the index $n$ can be made invariant also under nonglobally defined transformations if there is a gauge connection on $S^{2}$; this can be achieved by introducing an arbitrary one to enlarge the space of allowed transformations sufficiently. But our physical framework already possesses a natural candidate- the spatial gauge potential $\boldsymbol{A}$. Then, according to general theory, we can write

$$
n(g, A)=-\frac{1}{32 \pi} \int_{S^{2}} \operatorname{Tr} g\left[d_{A} g, d_{A} g\right]-\frac{1}{2 \pi} \int_{S^{2}} \operatorname{Tr}\left[g F_{A}\right],
$$

with $d_{A} g=d g+[\boldsymbol{A}, g]$ and $F_{A}=d \boldsymbol{A}+\frac{1}{2}[\boldsymbol{A}, \boldsymbol{A}]$ is the associated magnetic field. The invariance under nonsmooth transformation is manifest, since no integration by part is required to prove that Eq. (3.5) is gauge invariant. Rewriting it as

$$
n(g, \boldsymbol{A})=-\frac{1}{32 \pi} \int_{S^{2}} \operatorname{Tr} g[d g, d g]-\frac{1}{2 \pi} \int_{S^{2}} \operatorname{Tr}[d(g \boldsymbol{A})]
$$

shows that it is equivalent to Eq. (3.4) when $g$ and $\boldsymbol{A}$ are smooth, since the second integral in Eq. (3.23) vanishes.

Let us now perform the $S U(2)$ transformation that diagonalizes $g=h^{-1} \sigma_{3} h$; then

$$
n(g, \boldsymbol{A})=1=-\frac{1}{2 \pi} \int_{S^{2}} \operatorname{Tr}\left[\sigma_{3} d\left(\boldsymbol{A}^{h^{-1}}\right)\right]
$$

In particular, if we introduce the Abelian gauge connection $\boldsymbol{a} \equiv-\operatorname{Tr} \sigma_{3} A^{h^{-1}}$ we obtain

$$
n(g, A)=1=\frac{1}{2 \pi} \int_{S^{2}} d a
$$

$\boldsymbol{a}$ can indeed be interpreted as a $U(1)$ connection, since its transition functions are Abelian. The first Chern class of the component of $\boldsymbol{A}^{h^{-1}}$ along the Cartan subalgebra (here $\sigma_{3}$ ), is equal to the winding number of the original map. Geometrically, we have picked up a nontrivial torus bundle; physically, a nonvanishing magnetic flux, related to the diagonal components of the field, appears. We have thus associated to a $S U(2)$ connection a topological number that plays a dual role: in regular gauges it measures the obstruction to diagonalizing WPL, while in singular ones its presence results in the appearance of a magnetic flux. We have also learned how to move from one picture to its dual. The above discussion

\footnotetext{
${ }^{7}$ From the mathematical point of view we want to exploit the possibility of representing a given fiber bundle through any of the coordinate bundles belonging to its equivalence class. We call this - by a physicist's abuse of language - a gauge transformation, even though in mathematics that name is restricted to the vertical automorphism of the bundle.
}

naturally extends to any map $g$ from a two-dimensional manifold into the $S U(2)$ group. The generalization to $S U(N)$ does involve some subtleties; following Ref. [19], we therefore briefly summarize the fundamental steps. Given the WPL, patchwise we can always write

$$
\Omega=h_{\alpha}^{-1} s h_{\alpha}
$$

where $s$ is a diagonal matrix and $h_{\alpha}$ is map from the patch $U_{\alpha}$ into $S U(N)$. In each patch, we can also introduce another matrix $g_{\alpha}$,

$$
g_{\alpha}=h_{\alpha}^{-1} \mu h_{\alpha}
$$

where $\mu$ is a regular element in the Lie algebra of the maximal torus, an integer linear combination of the diagonal generators of $S U(N)$. One can show that $g$ is globally defined and thus drop the index $\alpha$; since $\Omega$ is assumed to be regular and since our $g$ commutes with $\Omega$, the obstruction to diagonalizing $\Omega$ is also carried by $g$. Choose $\mu$ to be one of the roots $\alpha^{k}$ of $S U(N)$. Then we can express $N-1$ integervalued indices $n^{k}$ describing the aforementioned obstruction in terms of $g$,

$$
n^{k}(g, \boldsymbol{A})=-\frac{1}{4 \pi} \int \operatorname{Tr} g[D(\boldsymbol{A}, g), D(\boldsymbol{A}, g)]-\frac{1}{2 \pi} \int \operatorname{Tr} g F_{A},
$$

where $D(\boldsymbol{A}, g)=\boldsymbol{A}-h^{-1} d h$. One might wonder why we introduced $g$, rather than working directly with $\Omega$. Briefly, global diagonalizability of a matrix is a property of the reference frame defined by its eigenvectors; since we consider regular maps, the explicit form of its eigenvalues is irrelevant and only their nondegeneracy matters. Therefore introducing $g$ is a simple tool for extracting only the relevant information. This also explains why we were able to give invariant meaning to the magnetic flux that emerged upon diagonalization: we have singled out a set of intrinsic vectors in the color space that transform covariantly under gauge transformations, and used them to bleach the color index, thus providing a (pseudo-)Abelian framework. ${ }^{8}$

The appearance of the new topological index and of the associated Abelian fluxes is an intriguing new feature, whose presence could affect the Dirac determinant effective action, especially its parity violating part. We have in mind analogous effects due to magnetic fields in $\mathrm{QED}_{3}$ or to instantons in $\mathrm{QCD}_{4}$. To quantify its repercussions, however, would require difficult calculations we have not attempted.

\footnotetext{
${ }^{8}$ If we try to diagonalize $g$, and consequently $\Omega$, it is not difficult to argue that, even in this more general $S U(N)$ case, the components of the connection along the Cartan subalgebra become nontrivial in the sense that they can acquire nonvanishing " $U(1)$ " Chern classes - one for each diagonal generator of $S U(N)$. For a more detailed discussion of this construction, see Ref. [19].
} 


\section{B. Examples}

We now proceed to illustrate the above general mechanism with two explicit examples, $S^{1} \times S^{2}$ and $S^{1} \times T^{2} \simeq T^{3}$. Consider first the following $S U(2)$ connection on $\mathcal{M}=S^{1}$ $\times S^{2}$,

$$
A_{0}(\theta, \phi)=\frac{2 \pi \gamma}{\beta}\left(\begin{array}{cc}
\cos \theta & \sin \theta e^{-i n \phi} \\
\sin \theta e^{i n \phi} & -\cos \theta
\end{array}\right), \quad \boldsymbol{A}=0
$$

it is globally defined when the angles $(\theta, \phi)$ span the unit sphere. Note that there is no magnetic flux through the sphere since the spatial components $\boldsymbol{A}$ vanish. ${ }^{9}$ What happens if we try to diagonalize the WPL, $\exp \left(i \beta A_{0}\right)$, associated with the above (time-independent) $A_{0}$ ? The diagonalizing transformation

$$
U_{n}(\theta, \phi)=\left(\begin{array}{cc}
\cos (\theta / 2) & -e^{-i n \phi} \sin (\theta / 2) \\
\sin (\theta / 2) e^{i n \phi} & \cos (\theta / 2)
\end{array}\right)
$$

is not globally defined on the sphere: for example, $\phi$ dependence clearly remains at the south pole, $\theta=\pi$. Hence this gauge transformation is defined only in the north pole chart of the sphere and another one, regular in the south pole chart, must be constructed in order to achieve consistent diagonalization. For the moment, let us work only around the north pole; it will be clear how to extend the final result to the south pole region. Performing Eq. (3.13) on Eq. (3.12) yields $A_{0}=2 \pi \gamma \sigma_{3} / \beta$, but also introduces space components:

$$
\begin{aligned}
& A_{\theta}^{U}=-\frac{i}{2}\left(\begin{array}{cc}
0 & e^{-i n \phi} \\
-e^{i n \phi} & 0
\end{array}\right), \\
& A_{\phi}^{U}=\frac{n}{2}\left(\begin{array}{cc}
2 \sin ^{2}(\theta / 2) & -e^{-i n \phi} \sin \theta \\
-e^{i n \phi} \sin \theta & -2 \sin ^{2}(\theta / 2)
\end{array}\right) .
\end{aligned}
$$

We can now construct the apparently Abelian field that keeps track of the hidden original obstruction to diagonalizing $A_{0}$, namely

$$
\begin{aligned}
& a_{\theta} \equiv \frac{1}{2} \operatorname{Tr}\left(\sigma_{3} A_{\theta}^{U}\right)=0 ; \\
& a_{\phi} \equiv \frac{1}{2} \operatorname{Tr}\left(\sigma_{3} A_{\phi}^{U}\right)=n \sin ^{2} \frac{\theta}{2}=\frac{n}{2}(1-\cos \theta) .
\end{aligned}
$$

\footnotetext{
${ }^{9} \mathrm{We}$ also remark that the constant $\gamma$ cannot be an integer, since then WPL becomes the identity, obviously not a regular map [it commutes with any element of $S U(2)]$. Physically, $\gamma$ plays the role of a flat connection and integer-valued $\gamma$ allow $A_{0}$ to be completely gauged away. We also mention that making $\gamma$ angle dependent does not alter the results below. Nothing essential would change either had we chosen $\boldsymbol{A}=\boldsymbol{A}(t, \boldsymbol{x})$ instead of $\boldsymbol{A}=0$. In fact, if $\boldsymbol{A}(t, \boldsymbol{x})$ is a globally defined connection on the sphere, its overall effect on the Abelian gauge field $\left(a_{\theta}, a_{\phi}\right)$ below is to add a topologically trivial fluctuation, given by $\operatorname{Tr}\left(A_{0} \boldsymbol{A}\right)$, that would not alter the value of the Chern class.
}

We immediately recognize the usual monopole on the twosphere, or more precisely, its usual expression in the north pole chart. The corresponding field strength is proportional to the volume form of the sphere and therefore carries a nonvanishing Chern class with value $n$. Analysis of a gauge transformation regular about the south pole yields the expression for the same monopole in that chart. As expected from general theory, the gauge transformation connecting the two expressions belongs to the maximal torus: it is proportional to $\sigma_{3}$. For completeness, we write the gauge invariant form of this index in terms of the above fields. If we call $g$ $=U_{n} \sigma_{3} U_{n}^{-1} / 2=\beta \Omega / 4 \pi \gamma$, then in any gauge, the index is

$$
n=-\frac{1}{32 \pi} \int_{S^{2}} \operatorname{Tr}\left(g\left[d_{A} g, d_{A} g\right]\right),
$$

which is the same as Eq. (3.5) upon dropping the magnetic field term, since $B$ vanishes in all gauges. Note incidentally that, being gauge invariant, Eq. (3.16) could appear in the effective action for such backgrounds.

It is instructive to consider an example defined on a different 2-surface, the 2-torus $T^{2}$, so that now $\mathcal{M}=S^{1} \times T^{2}$ $\simeq T^{3}$. The spatial torus $T^{2}$ is parametrized by two flat periodic coordinates $x_{1} \sim x_{1}+1$ and $x_{2} \sim x_{2}+1$. We start by considering a transformation $V\left(x_{1}, x_{2}\right)$ of $S U(2)$ periodic up to an element $\omega_{i}$ of the maximal torus; in particular we choose

$$
V\left(x_{1}+1, x_{2}\right)=\omega_{1} V\left(x_{1}, x_{2}\right)=\left(\begin{array}{cc}
e^{-2 \pi i x_{2}} & 0 \\
0 & e^{2 \pi i x_{2}}
\end{array}\right) V\left(x_{1}, x_{2}\right),
$$

$V\left(x_{1}, x_{2}+1\right)=\omega_{2} V\left(x_{1}, x_{2}\right)=V\left(x_{1}, x_{2}\right)$,

where the explicit form of $\omega_{i}$ is the trivial embedding in $S U(2)$ of the transition functions of the $U(1)$ instanton with unit charge on $T^{2}$. We can now construct a globally defined (i.e., periodic) WPL as follows:

$$
\begin{aligned}
\Omega\left(x_{1}, x_{2}\right)= & V^{-1}\left(x_{1}, x_{2}\right) \\
& \times\left(\begin{array}{cc}
\exp \left(i \phi\left(x_{1}, x_{2}\right)\right) & 0 \\
0 & \exp \left(-i \phi\left(x_{1}, x_{2}\right)\right)
\end{array}\right) \\
& \times V\left(x_{1}, x_{2}\right) \\
= & \left(\begin{array}{cc}
|\alpha|^{2} e^{i \phi}+|\beta|^{2} e^{-i \phi} & 2 i \bar{\alpha} \bar{\beta} \sin \phi \\
-2 i \alpha \beta \sin \phi & e^{i \phi}|\beta|^{2}+|\alpha|^{2} e^{-i \phi}
\end{array}\right),
\end{aligned}
$$

in terms of the parametrization

$$
V\left(x_{1}, x_{2}\right)=\left(\begin{array}{cc}
\alpha\left(x_{1}, x_{2}\right) & \bar{\beta}\left(x_{1}, x_{2}\right) \\
-\beta\left(x_{1}, x_{2}\right) & \bar{\alpha}\left(x_{1}, x_{2}\right)
\end{array}\right), \quad|\alpha|^{2}+|\beta|^{2}=1
$$

The eigenvalues $\exp ( \pm i \phi)$ of $\Omega$ are taken to be periodic. The periodicity, and consequently the global nature of $\Omega$, instead follows from the fact that the transformation $\omega_{i}$ belongs to the maximal torus. 
Now we show that attempting to diagonalize the above WPL gives rise to a magnetic flux. The additional components along the Cartan subalgebra are, as already explained in the previous example,

$$
A_{i}\left(x_{1}, x_{2}\right)=\frac{1}{2} \operatorname{Tr}\left[\sigma_{3}\left(\partial_{i} V\left(x_{1}, x_{2}\right)\right) V^{-1}\left(x_{1}, x_{2}\right)\right] .
$$

They satisfy the following periodicity conditions

$$
\begin{aligned}
A_{1}\left(x_{1}+1, x_{2}\right) & =\frac{1}{2} \operatorname{Tr}\left[\sigma_{3}\left(\partial_{1} V\left(x_{1}+1, x_{2}\right)\right) V^{-1}\left(x_{1}+1, x_{2}\right)\right] \\
& =A_{1}\left(x_{1}, x_{2}\right), \\
A_{2}\left(x_{1}+1, x_{2}\right) & =\frac{1}{2} \operatorname{Tr}\left[\sigma_{3}\left(\partial_{2} V\left(x_{1}+1, x_{2}\right)\right) V^{-1}\left(x_{1}+1, x_{2}\right)\right] \\
& =A_{1}\left(x_{1}, x_{2}\right)-2 \pi, \\
\boldsymbol{A}\left(x_{1}, x_{2}+1\right) & =\boldsymbol{A}\left(x_{1}, x_{2}+1\right) .
\end{aligned}
$$

This is exactly the behavior of a (unit charge) instanton on $T^{2}$. We thus can say equivalently that this field carries a magnetic flux equal to 1 or, in the regular gauge, that WPL defines a mapping with unit winding number. Finally, we must demonstrate that a matrix $V\left(x_{1}, x_{2}\right)$ satisfying Eq. (3.17) indeed exists; in the parametrization $(3.20),(\alpha, \beta)$ must obey

$$
\begin{aligned}
& \alpha\left(x_{1}+1, x_{2}\right)=\exp \left(-2 \pi i x_{2}\right) \alpha\left(x_{1}, x_{2}\right), \\
& \alpha\left(x_{1}, x_{2}+1\right)=\alpha\left(x_{1}, x_{2}\right), \\
& \beta\left(x_{1}+1, x_{2}\right)=\exp \left(2 \pi i x_{2}\right) \beta\left(x_{1}, x_{2}\right), \\
& \beta\left(x_{1}, x_{2}+1\right)=\beta\left(x_{1}, x_{2}\right) .
\end{aligned}
$$

These periodicity requirements are solved in terms of $\Theta$ functions, namely by

$$
\alpha\left(x_{1}, x_{2}\right)=\frac{1}{\mathcal{N}} \bar{\Theta}\left[\begin{array}{l}
x_{2} \\
x_{1}
\end{array}\right](0, i) \quad \beta\left(x_{1}, x_{2}\right)=\frac{1}{\mathcal{N}} \Theta\left[\begin{array}{c}
x_{2} \\
x_{1}+\lambda
\end{array}\right](0, i)
$$

where the normalizing factor $\mathcal{N}$ maintains the condition $|\alpha|^{2}+|\beta|^{2}=1$ and $\lambda$ is any (noninteger) constant. Our construction also suggests a simple and natural representative LGT on $T^{3}$, namely

$$
U_{n}\left(t, x_{1}, x_{2}\right)=V^{-1}\left(x_{1}, x_{2}\right) e^{i \pi n t \sigma_{3} / \beta} V\left(x_{1}, x_{2}\right) e^{-i \pi n t \sigma_{3} / \beta} ;
$$

it is globally defined on $T^{3}$ and has winding number $n$. (A similar construction in a $\mathrm{QCD}_{4}$ context was examined in Ref. [16].)

\section{THE FATE OF LGT}

Throughout the literature $[1,3,6,9,10,14,15]$ on effective non-Abelian actions, a difficult question, as we have already mentioned, has been the role and form of LGT, a name indiscriminately attributed to two different classes of transformations. The first is that (discussed in Sec. II) of the usual color LGT with nonvanishing $W_{2}(U)$. The second is that of the "Abelian" LGT, whose action, in the gauge where the WPL is diagonal, is to shift the flat connection wrapping around the Euclidean time direction by an integer-valued diagonal matrix. In this gauge, it has the simple form

$$
u(t)=\exp \left(\frac{2 \pi i t}{\beta} D\right)
$$

where $D$ is a linear integer combination of the diagonal generators of $S U(N)$. In most explicit computations, the question of the invariance of the finite temperature effective actions under LGT refers to this second class. These transformations, as is manifest when written in the form (4.1), can be unwrapped smoothly, hence they cannot be considered a subset of the actual LGT. Calling them "LGT" at this level merely reflects their formal resemblance to the Abelian ones. They are in fact LGT for the subgroup $U(1)^{N-1}$, but they seemingly lose this property when immersed in $S U(N)$ : their winding number $W_{2}(u)$, when computed from Eq. (4.1), vanishes. In the following, we will show that they are nevertheless LGT in a deeper sense: The "Abelian" LGT appearing in the gauge where the WPL is diagonal and a magnetic flux is present become, in the dual picture where all the fields are made regular, transformations which are genuinely large $\left(W_{2} \neq 0\right)$. This dualism between large and small transformations is the manifestation of a more general problem: the meaning and the definition of LGT when we deal with nontrivial bundles, or more generically, with bundles whose transition functions are not the identity. In fact, the index $W_{2}(U)$ in the form (2.15) is not sufficient to capture the winding of a transformation when we use a nonglobal section of the gauge bundle. For us, this problem was not dramatic since we just considered $S U(N)$, which admit only trivial bundles and thus we always had a global section from which to check if a transformation is large. This problem becomes unavoidable, however, for nonsimply connected group or dimensions different from three; one such case was indeed briefly discussed in Sec. II.

It is convenient here to select the well-known (almost) temporal gauge

$$
\dot{A}_{0} \equiv \partial_{0} A_{0}=0 \Leftrightarrow A_{0}(t, x) \equiv \frac{2 \pi}{\beta} A_{0}(x) ;
$$

periodicity prevents one from setting $A_{0}=0$, since this would entail a trivial WPL. Investigating the role of LGT when we have already picked a specific gauge might appear self-contradictory, but our choice leaves a residual freedom that includes representatives of large gauge transformations. The fact that this gauge is attainable is shown for example in Ref. [34]. Here we briefly review the main steps of its construction since they are necessary in understanding the residual gauge group. Imposing the gauge (4.2) amounts to finding periodic solutions of the linear differential equation 


$$
\begin{gathered}
\partial_{t} U(t, \boldsymbol{x})+i A_{0}(t, \boldsymbol{x}) U(t, \boldsymbol{x})=\frac{2 \pi i}{\beta} U(t, \boldsymbol{x}) \mathrm{A}_{0}(\boldsymbol{x}), \\
U(\beta, \boldsymbol{x})=U(0, \boldsymbol{x}) .
\end{gathered}
$$

In terms of the new variable $V(t, x)=\Omega^{-1}(t, x) U(t, x)$, Eq. (4.3) reduces to the homogeneous equation,

$$
\partial_{t} V(t, \boldsymbol{x})=\frac{2 \pi i}{\beta} V(t, \boldsymbol{x}) \mathrm{A}_{0}(\boldsymbol{x}),
$$

since $\quad \Omega(t, \boldsymbol{x})=P \exp \left[-i \int_{0}^{t} d t^{\prime} A_{0}\left(t^{\prime}, \boldsymbol{x}\right)\right] \quad$ obeys $\quad \partial_{t} \Omega(t, \boldsymbol{x})$ $+i A_{0}(t, \boldsymbol{x}) \Omega(t, \boldsymbol{x})=0$. Since $\mathrm{A}_{0}(\boldsymbol{x})$ is time independent, Eq. (4.4) can be promptly integrated,

$$
V(t, \boldsymbol{x})=\hat{V}(\boldsymbol{x}) \exp \left(\frac{2 \pi i t}{\beta} \mathrm{A}_{0}(\boldsymbol{x})\right) .
$$

Thus the general solution of Eq. (4.3) is

$$
U(t, \boldsymbol{x})=\Omega(t, \boldsymbol{x}) \hat{V}(\boldsymbol{x}) \exp \left(\frac{2 \pi i t}{\beta} \mathrm{A}_{0}(\boldsymbol{x})\right) .
$$

Imposing the periodicity condition $U(\beta, x)=U(0, x)$ determines the possible values $A_{0}(\boldsymbol{x})$ in terms of WPL, according to

$$
\hat{V}^{-1}(\boldsymbol{x}) \Omega(\beta, \boldsymbol{x}) \hat{V}(\boldsymbol{x})=\exp \left[-2 \pi i \mathrm{~A}_{0}(\boldsymbol{x})\right],
$$

i.e., $A_{0}$ is essentially the logarithm of WPL. Whether this logarithm always defines a global quantity is not obvious; however one can see easily that, at least for regular maps, it does. (Even in the general case this logarithm can actually be safely taken, but establishing that requires more careful analysis.)

We now probe the residual gauge freedom remaining after the choice (4.2); Eq. (4.6) immediately implies that any transformation of the form

$$
U(t, \boldsymbol{x})=\exp \left(-\frac{2 \pi i t}{\beta} \mathrm{A}_{0}(\boldsymbol{x})\right) \hat{V}(\boldsymbol{x}) \exp \left(\frac{2 \pi i t}{\beta} \mathrm{B}_{0}(\boldsymbol{x})\right),
$$

preserves Eq. (4.2) if

$$
\hat{V}^{-1}(\boldsymbol{x}) \exp \left[-2 \pi i \mathrm{~A}_{0}(\boldsymbol{x})\right] \hat{V}(\boldsymbol{x})=\exp \left[-2 \pi i \mathbb{B}_{0}(\boldsymbol{x})\right],
$$

which is just Eq. (4.7), but only within the class of gaugeequivalent fields that respect Eq. (4.2). Let us change variables from $\mathbb{B}_{0}(\boldsymbol{x})$ to $\Lambda(\boldsymbol{x})$ by means of

$$
\mathbb{B}_{0}(\boldsymbol{x})=\hat{V}^{-1}(\boldsymbol{x})\left[\mathrm{A}_{0}(\boldsymbol{x})+\Lambda(\boldsymbol{x})\right] \hat{V}(\boldsymbol{x}) .
$$

This final shift to the variable $\Lambda$ then leads to the simple but powerful statement

$$
\exp \left[2 \pi i \mathrm{~A}_{0}(\boldsymbol{x})\right]=\exp \left\{2 \pi i\left[\mathrm{~A}_{0}(\boldsymbol{x})+\Lambda(\boldsymbol{x})\right]\right\} .
$$

If $\exp \left[2 \pi i \mathrm{~A}_{0}(\boldsymbol{x})\right]$ is a regular map, this implies, for all $\boldsymbol{x}$, the two conditions

$$
\left[\exp \left[2 \pi i \mathrm{~A}_{0}(\boldsymbol{x})\right], \Lambda(\boldsymbol{x})\right]=0, \quad \exp [2 \pi i \Lambda(\boldsymbol{x})]=1,
$$

so the eigenvalues of $\Lambda$ are integers. We can thus conclude that the most general transformation that preserves our gauge choice is

$$
U(t, x)=\exp \left(\frac{2 \pi i}{\beta} t \Lambda(\boldsymbol{x})\right) \hat{V}(\boldsymbol{x}) .
$$

Now it is immediate to recognize a connection with the previous section: $\Lambda(\boldsymbol{x})$ is nothing but the (properly normalized) auxiliary function $g(\boldsymbol{x})$ introduced in Eq. (3.10). More generally it could be any integer combination of the $g$ associated to the different roots. Thus if $A_{0}(\boldsymbol{x})$ corresponds to a field whose WPL is not smoothly diagonalizable, the function $\Lambda(\boldsymbol{x})$ can be chosen to carry this information, through having a nontrivial index. As a consequence,

$$
U(t, \boldsymbol{x})=\exp \left(\frac{2 \pi i}{\beta} t \Lambda(\boldsymbol{x})\right)
$$

preserves the gauge (4.2); it is both LGT and periodic. The LGT property follows from the fact the $\Lambda(x)$ is not smoothly diagonalizable. In fact, recalling that $\Lambda(\boldsymbol{x})$ has been identified with $g(\boldsymbol{x})=h^{-1} \alpha h$, we can write

$$
\partial_{t} U=\frac{2 \pi i}{\beta} U \Lambda, \quad \partial_{i} U=\left[U, h^{-1} \partial_{i} h\right] .
$$

In turn, the index of the transformation can be recast as

$$
\begin{aligned}
W_{2}(U)= & \frac{i}{8 \pi \beta} \int_{0}^{\beta} d t \int_{\Sigma} d x \epsilon^{i j} \\
& \times \operatorname{Tr}\left(\Lambda\left[U^{-1}\left[U, h^{-1} \partial_{i} h\right], U^{-1}\left[U, h^{-1} \partial_{j} h\right]\right]\right) .
\end{aligned}
$$

It is easy to see that the integrand in $W_{2}$ is the sum of two terms,

$$
\begin{aligned}
& X \equiv \epsilon^{i j} \operatorname{Tr} \Lambda\left[h^{-1} \partial_{i} h, h^{-1} \partial_{j} h\right], \\
& Y \equiv \epsilon^{i j} \operatorname{Tr} \Lambda\left[h^{-1} \partial_{i} h, U^{-1} h^{-1} \partial_{j} h\right],
\end{aligned}
$$

and that $Y$ is a total time derivative whose integral vanishes by periodicity, while $X$ is time independent. Hence, it easily follows that

$$
W_{2}(U)=1 / 4 \pi \int d^{2} x X=2 n_{2}(\Lambda) .
$$

But $n_{2}(\Lambda)$ is precisely the value, at $\boldsymbol{A}=0$, of the index defined in Eq. (3.11). This finally proves that the residual group for nondiagonalizable WPL still contains (the "true") LGT. Of course, $\Lambda$ is neither fixed nor unique: there are in general $N-1$ linearly independent matrices $\Lambda^{i}$ that commute with $A_{0}$. Therefore we can construct, in principle, a wide array of transformations by taking integer linear combinations of $\Lambda^{i}$. Unfortunately, this space does not contain all the large gauge 
transformations, but just those whose index is an even multiple of the flux associated to the torus bundle. However, their action on $A_{0}$ is simply the shift

$$
A_{0} \rightarrow A_{0}+2 \pi \Lambda .
$$

When we diagonalize the WPL (and consequently $A_{0}$ ), $\Lambda$ also diagonalizes since it commutes with $A_{0}$. In terms of these diagonal forms $\left(a_{0}\right)$, the above equation reduces to

$$
a_{0} \rightarrow a_{0}+2 \pi D,
$$

where $D$ is an integer linear combination of the diagonal generators of $S U(N)$. These transformations can be interpreted to act on the constant part of the eigenvalues of $a_{0}$; but this is exactly the result of a transformation of the form (4.1).

Summarizing, when we gauge diagonalize WPL (which does not alter our gauge condition $\dot{A}_{0}=0$ ), the surviving LGT are further reduced to the simpler form (4.1). This is, in a certain sense, expected because we limit our gauge functions to those of the torus bundle in the process and the surviving LGT share this fate: They become LGT of the torus bundle gauge group $U(1)^{N-1}$. When the fluxes vanish, obviously no large transformation survives the chosen gauge fixing. In this limit, the flat connection becomes essentially irrelevant and nontrivial interplays between topological quantities in the magnetic sector are absent. Only the (topologically dull) electric sector remains.

\section{VANISHING $E$}

The final example in Sec. III illustrated the seemingly paradoxical extreme situation of starting with no magnetic field and ending up with a field on the maximal torus that actually carries a flux. In the recent literature $[9,14,15]$ on explicit calculations of effective actions, the opposite extreme has also been considered, where instead, $\boldsymbol{E}$ vanished. In the discussions of the general structure of these latter configurations, a certain amount of confusion has arisen, whose origin lies in nongauge invariant descriptions of the fluxes discussed here. Hence we next review the general form of such fluxes.

Since the vanishing of $\boldsymbol{E}$ is a gauge invariant property, we simplify our analysis by choosing the gauge of Sec. IV, and henceforth assume that $A_{0}(t, \boldsymbol{x})=\mathrm{A}_{0}(\boldsymbol{x})$; the latter is not arbitrary however, since $\Omega=\exp \left[2 \pi i \mathrm{~A}_{0}(\boldsymbol{x})\right]$, and $\Omega$ is constrained to be covariantly constant $[35,36]$,

$$
D_{i} \Omega \equiv \partial_{i} \Omega+\left[A_{i}(0, \boldsymbol{x}), \Omega\right]=0 .
$$

Its gauge invariant content can be understood by taking the derivative of $\operatorname{Tr}\left(\Omega^{n}\right)$ :

$$
\partial_{i}\left[\operatorname{Tr}\left(\Omega^{n}\right)\right]=n \operatorname{Tr}\left(\Omega^{n-1} \partial_{i} \Omega\right)=n \operatorname{Tr}\left(\Omega^{n-1} D_{i} \Omega\right)=0,
$$

i.e., the trace of any power of $\Omega$ is independent of $\boldsymbol{x}$, and its eigenvalues are all constant. Similarly, the Bianchi identities imply that the magnetic field $B \equiv F_{12}$ is covariantly conserved,

$$
D_{t} B(t, \boldsymbol{x})=\partial_{t} B(t, \boldsymbol{x})+i\left(\mathrm{~A}_{0}(\boldsymbol{x}), B(t, \boldsymbol{x})\right)=0,
$$

or equivalently,

$$
\partial_{t}\left[\exp \left(-\frac{2 \pi i t}{\beta} \mathrm{A}_{0}(\boldsymbol{x})\right) B(t, \boldsymbol{x}) \exp \left(\frac{2 \pi i t}{\beta} \mathrm{A}_{0}(\boldsymbol{x})\right)\right]=0 .
$$

When integrated between $(0, t)$, this yields

$$
B(t, \boldsymbol{x})=\exp \left(\frac{2 \pi i t}{\beta} \mathrm{A}_{0}(\boldsymbol{x})\right) B(0, \boldsymbol{x}) \exp \left(-\frac{2 \pi i t}{\beta} \mathrm{A}_{0}(\boldsymbol{x})\right) .
$$

This does not yet imply that $B(t, \boldsymbol{x})$ can be made time independent, because the WPL does not define a legitimate, periodic, gauge transformation ${ }^{10}$ still. The periodicity condition $B(0, x)=B(\beta, x)$ does require the WPL to commute with $B(0, \boldsymbol{x})$,

$$
\left[B(0, \boldsymbol{x}), \exp \left[2 \pi i \mathrm{~A}_{0}(\boldsymbol{x})\right]\right]=0 .
$$

In the subsequent we limit ourselves to regular WPL (recall again that a regular map means, for $S U(N)$, that all its eigenvalues are different); most of the result will not depend on this technical assumption; we shall comment further on this point. Then Eq. (5.6) immediately entails that $\left(\mathrm{A}_{0}, B(0, \boldsymbol{x})\right)=0$, which, combined with Eq. (5.5), is equivalent to the time independence of $B$.

So far, we have seen that one we can always achieve a gauge where $A_{0}$ and $B$ are both time independent and commute. The following steps become much easier if we further diagonalize $A_{0}$ and $B$, as can be always arranged because the residual gauge freedom contains all time-independent transformations. The price is that the components of $\boldsymbol{A}$ along the Cartan subalgebra might live on a nontrivial torus bundle, but we have learned how to deal with this difficulty in Sec. III. Thus, from now on we have, in our "torus gauge,"

$$
\mathrm{A}_{0}(\boldsymbol{x})=a_{0}^{i} H_{i} \quad \text { and } \quad B(\boldsymbol{x})=\mathcal{B}^{i}(\boldsymbol{x}) H_{i},
$$

where the $H_{i}$ generate the Cartan subalgebra. The factor $a_{0}^{i}$ are constant, being linear combinations of the eigenvalues of the WPL. The vanishing of $\boldsymbol{E}$ allows us to make both $B$ and $\boldsymbol{A}$ time independent. In fact, in our diagonal gauge, this condition $\left(\boldsymbol{E} \equiv \partial_{t} \boldsymbol{A}+\left[a_{0}, \boldsymbol{A}\right]=0\right)$ can be easily integrated between $(0, t)$ to yield

$$
\boldsymbol{A}(t, \boldsymbol{x})=\exp \left(-\frac{2 \pi i t}{\beta} a_{0}\right) \boldsymbol{A}(0, \boldsymbol{x}) \exp \left(\frac{2 \pi i t}{\beta} a_{0}\right) .
$$

This has the same structure as Eq. (5.5) and, by the same arguments, leads to the analogous conclusion,

$$
\left[a_{0}, \boldsymbol{A}(0, \boldsymbol{x})\right]=0,
$$

\footnotetext{
${ }^{10}$ We allow only periodic transformations since for the moment we want to have globally defined potentials, namely ones that do not differ by a gauge transformation when we change charts on $S^{1}$.
} 
and consequently to time independence, $\boldsymbol{A}(t, \boldsymbol{x})=\boldsymbol{A}(0, \boldsymbol{x})$.

Summarizing, for vanishing electric field, if the WPL is a regular map, we can always choose a gauge where all connections are time independent, $A_{0}$ is also space independent and finally $\left[A_{0}, \boldsymbol{A}\right]=0$. This configuration can carry a magnetic flux. ${ }^{11}$ In particular our result shows that if $\boldsymbol{E}=0$, a magnetic flux must be present for there at all to be a nontrivial effective action in the PV sector: otherwise the candidate $\mathrm{CS}$ action, $\int \operatorname{Tr}\left(a_{0} B\right)$, vanishes.

We now briefly comment on non regular maps. In general, this question is very involved, as one must know the general structure of the submanifold where $\Omega$ has degenerate eigenvalues. For us, $\boldsymbol{E}=0$ provides a substantial simplification; the eigenvalues do not depend on $\boldsymbol{x}$, and therefore the presence of degenerate ones is also $\boldsymbol{x}$ independent. This protects us from pathologies such as permutations of the eigenvalues arising from their flow through the spatial surface, so our results persist also in this context.

\section{SUMMARY}

We have studied the interplay between color (already present at $T=0$ ) and thermal LGT, and the consequences of the ensuing topological complications. The central quantity that carries the large information is the WPL, generalizing the flat connection of the simple Abelian $U(1)$. We provided the relevant mathematical framework for keeping track of the

\footnotetext{
${ }^{11}$ It has been claimed [15] that there exist configurations with vanishing $\boldsymbol{E}$ that are not gauge equivalent to those obtained above. In particular, it was stated that there are, in this class, connections that are genuinely time dependent. However, two points were overlooked: First, the possibility of diagonalizing $A_{0}$ through residual gauge transformations was not exploited. Second, only globally defined gauge transformations were allowed, a requirement we saw to be too restrictive once nontrivial torus bundles were admitted.
}

mapping involved in this "double LGT" world. We then exhibited explicit nonsingular LGT, studied their effects on WPL, and stressed the novel index that measures topological obstruction to its global diagonalization. We also linked the index to extension of the usual global WPL diagonalization process with a wider class of transformations that are not globally defined, but require use of local charts. Our explicit constructions, besides the mentioned LGT example, included the gauge potentials in temporal gauge for configurations with nonvanishing index. Then, for vanishing $\boldsymbol{E}$ (that we do not believe to be an essential restriction), we showed precisely how "magnetism without magnetism" arises, i.e., how transforming configurations with vanishing $B$ gives rise to gauge invariant magnetic flux properties in the Cartan subalgebra directions. In the process, we corrected some misconceptions in the recent literature.

The clarifications we hope to have provided here should be thought of as an entry to a number of issues we have left untouched. To mention a few, the effect of an index on the parity-violating parts of the effective action/Dirac determinant might be quite extensive; perhaps it could be probed using some simple but indicative special configurations. Any fallout to nonperturbative $D=4$ effects would of course be particularly important, but may be more remote in view of the pivotal role of odd dimension in our considerations. Finally, the "kinematics" we have attempted to sort out should be of use in a more explicit analysis of $\mathrm{QCD}_{3}$ dynamics, perhaps without recourse to particular field configurations.

\section{ACKNOWLEDGMENTS}

This work was supported by the National Science Foundation under grant PHY99-73935; D.S. was supported by TMR grant FMRX CT96-0045. D.S. and L.G. were supported by the Bruno Rossi program during the final phase of this work, begun in the previous millennium; both thank MIT for its warm hospitality. We thank C. Isham for useful correspondence.
[1] S. Deser, L. Griguolo, and D. Seminara, Phys. Rev. Lett. 79, 1976 (1997); Phys. Rev. D 57, 7444 (1998).

[2] S. Deser, L. Griguolo, and D. Seminara, Commun. Math. Phys. 197, 443 (1998).

[3] A.J. Niemi, Nucl. Phys. B251, 155 (1985); A.N. Redlich and L.C.R. Wijewardhana, Phys. Rev. Lett. 54, 970 (1985); A.J. Niemi and G.W. Semenoff, ibid. 54, 2166 (1985).

[4] R.D. Pisarski, Phys. Rev. D 35, 664 (1987).

[5] K.S. Babu, A. Das, and P. Panigrahi, Phys. Rev. D 36, 3725 (1987); I.J.R. Aitchison, C.D. Fosco, and J.A. Zuk, ibid. 48, 5895 (1993); I.J. Aitchison and J.A. Zuk, Ann. Phys. (N.Y.) 242, 77 (1995).

[6] G. Dunne, K. Lee, and C. Lu, Phys. Rev. Lett. 78, 3434 (1997)

[7] A. Das and G. Dunne, Phys. Rev. D 57, 5023 (1998); A.K. Das, G.V. Dunne, and J. Frenkel, Phys. Lett. B 472, 332 (2000)

[8] C. Fosco, G.L. Rossini, and F.A. Schaposnik, Phys. Rev. Lett.
79, 1980 (1997); 79, 4296(E) (1997).

[9] L.L. Salcedo, Nucl. Phys. B549, 98 (1999); Phys. Lett. B 530, 244 (2002).

[10] F.T. Brandt, A. Das, and J. Frenkel, Phys. Rev. D 62, 085012 (2000).

[11] S. Deser, R. Jackiw, and S. Templeton, Phys. Rev. Lett. 48, 975 (1982); Ann. Phys. (N.Y.) 140, 372 (1982).

[12] L. Alvarez-Gaumé, S. Della Pietra, and G.W. Moore, Ann. Phys. (N.Y.) 163, 288 (1985).

[13] H. Leutwyler, Helv. Phys. Acta 63, 660 (1990).

[14] C.D. Fosco, G.L. Rossini, and F.A. Schaposnik, Phys. Rev. D 56, 6547 (1997).

[15] F.T. Brandt, A. Das, and J. Frenkel, Phys. Rev. D 65, 065013 (2002).

[16] C. Ford, U.G. Mitreuter, T. Tok, A. Wipf, and J.M. Pawlowski, Ann. Phys. (N.Y.) 269, 26 (1998).

[17] G. 't Hooft, Nucl. Phys. B190, 455 (1981); H.W. Griesshammer, "Magnetic Defects Signal Failure of Abelian Projection 
Gauges in QCD," hep-ph/9709462; C. Ford, T. Tok, and A. Wipf, Phys. Lett. B 456, 155 (1999); P. Van Baal, Nucl. Phys. B (Proc. Suppl.) 108, 3 (2002).

[18] For a review, see H.W. Griesshammer, Ph.D. thesis, Universität Erlangen-Nürnberg, 1996; F. Lenz, H.W. Naus, and M. Thies, Ann. Phys. (N.Y.) 233, 317 (1994).

[19] M. Blau and G. Thompson, Nucl. Phys. B408, 345 (1993); Commun. Math. Phys. 171, 639 (1995).

[20] R. Dijkgraaf and E. Witten, Commun. Math. Phys. 129, 393 (1990).

[21] N. Steenrod, The Topology of Fiber Bundles (Princeton University Press, Princeton, NJ, 1951).

[22] S.J. Avis and C.J. Isham, in Recent Advances in Gravitation, edited by S. Deser and M. Levy (Plenum, New York, 1979).

[23] C.J. Isham, in Essays in Honour of Wolfgang Yourgrau, edited by A. van der Merwe (Plenum, New York, 1981).

[24] J.W. Vick, Homology Theory (Academic Press, New York, 1973).
[25] D. Husemoller, Fibre Bundles, 3rd ed. (Springer Verlag, New York, 1994).

[26] R. Jackiw and C. Rebbi, Phys. Rev. Lett. 37, 172 (1976); C.G. Callan, R.F. Dashen, and D.J. Gross, Phys. Lett. 63B, 334 (1976).

[27] N.K. Pak and R. Percacci, Nucl. Phys. B188, 355 (1981).

[28] F.T. Brandt, A. Das, G.V. Dunne, J. Frenkel, and J.C. Taylor, Phys. Rev. D 65, 065006 (2002).

[29] A. Polychronakos, Nucl. Phys. B281, 241 (1987).

[30] R. Jackiw and S.Y. Pi, Phys. Lett. B 423, 364 (1998).

[31] O. Alvarez, Commun. Math. Phys. 100, 279 (1985).

[32] G. Felder, K. Gawedzki, and A. Kupiainen, Commun. Math. Phys. 117, 127 (1988).

[33] D. Gepner and E. Witten, Nucl. Phys. B278, 493 (1986).

[34] E. Langmann, M. Salmhofer, and A. Kovner, Mod. Phys. Lett. A 9, 2913 (1994).

[35] D.J. Gross, R.D. Pisarski, and L.G. Yaffe, Rev. Mod. Phys. 53, 43 (1981).

[36] P. Menotti and D. Seminara, Ann. Phys. (N.Y.) 240, 203 (1995). 